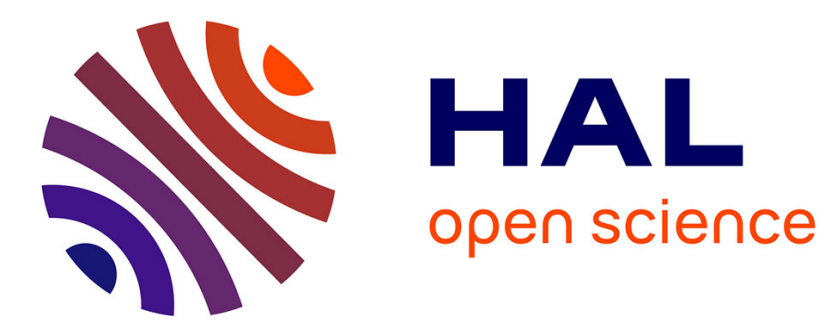

\title{
Contre-stéréotypes de sexe et littérature de jeunesse.
} Laurence Reeb, Christine Morin-Messabel, Kalampalikis Nikos

\section{To cite this version:}

Laurence Reeb, Christine Morin-Messabel, Kalampalikis Nikos. Contre-stéréotypes de sexe et littérature de jeunesse.. Bulletin de psychologie, 2018, 556 (4), pp.727-737. 10.3917/bupsy.556.0727 . halshs-01779957

\section{HAL Id: halshs-01779957 \\ https://shs.hal.science/halshs-01779957}

Submitted on 2 Jan 2019

HAL is a multi-disciplinary open access archive for the deposit and dissemination of scientific research documents, whether they are published or not. The documents may come from teaching and research institutions in France or abroad, or from public or private research centers.
L'archive ouverte pluridisciplinaire HAL, est destinée au dépôt et à la diffusion de documents scientifiques de niveau recherche, publiés ou non, émanant des établissements d'enseignement et de recherche français ou étrangers, des laboratoires publics ou privés. 


\section{CONTRE-STÉRÉOTYPES DE SEXE ET LITTÉRATURE DE JEUNESSE Laurence Reeb, Christine Morin-Messabel, Nikos Kalampalikis}

Groupe d'études de psychologie | «Bulletin de psychologie »

2018/4 Numéro 556 | pages 727 à 737

ISSN 0007-4403

Article disponible en ligne à l'adresse :

https://www.cairn.info/revue-bulletin-de-psychologie-2018-4-page-727.htm

\section{Pour citer cet article :}

Laurence Reeb et al., "Contre-stéréotypes de sexe et littérature de jeunesse 》, Bulletin de psychologie 2018/4 (Numéro 556), p. 727-737.

DOI 10.3917/bupsy.556.0727

Distribution électronique Cairn.info pour Groupe d'études de psychologie.

(C) Groupe d'études de psychologie. Tous droits réservés pour tous pays.

La reproduction ou représentation de cet article, notamment par photocopie, n'est autorisée que dans les limites des conditions générales d'utilisation du site ou, le cas échéant, des conditions générales de la licence souscrite par votre établissement. Toute autre reproduction ou représentation, en tout ou partie, sous quelque forme et de quelque manière que ce soit, est interdite sauf accord préalable et écrit de l'éditeur, en dehors des cas prévus par la législation en vigueur en France. Il est précisé que son stockage dans une base de données est également interdit. 


\title{
Contre-stéréotypes de sexe et littérature de jeunesse
}

\author{
REEB Laurence ${ }^{a}$ \\ Morin-Messabel Christine ${ }^{a}$ \\ KALAMPALIKIS Nikos ${ }^{a}$
}

a Université Lumière Lyon 2, Groupe de recherche en psychologie sociale (GRePS) - EA 4163, France.

Correspondance : Laurence Reeb, Université Lumière Lyon 2, GRePS, Institut de psychologie, 5 avenue Pierre Mendès-France, 69676 Bron, France.

Courriel : laurence.reeb@univ-lyon2.fr

Texte reçu le 27 avril 2017 et accepté le 12 février 2018.

http://www.bulletindepsychologie.net
Résumé : La littérature de jeunesse est un support privilégié d'acquisition des modèles sexués. L'objectif de notre recherche est d'étudier l'impact de contrestéréotypes de sexe présentés à travers des modèles auprès de 127 enfants de CE2-CM1 (63 filles et 64 garçons), dans le cadre de la littérature de jeunesse. Un questionnaire exploratoire a mesuré le lien avec le personnage et des éléments intentionnels faisant suite à l'histoire. Les résultats révèlent des scores supérieurs des filles par rapport aux garçons, et ce, pour toutes les questions. Des éléments que nous discutons et qu'il conviendra d'approfondir par la suite nous amènent à penser la nécessité de travailler sur les contre-stéréotypes de sexe auprès des enfants, de manière différenciée entre les filles et les garçons.

\section{Gender counter-stereotypes and children's literature}

Abstract: Children's literature is a favored medium for the acquisition of gender role models. The goal of our research is to study the impact of gender counter-stereotypes with 127 third- and fourth-grade children (63 girls and 64 boys), presented through role models within the framework of children's literature. An exploratory questionnaire assesses the relationship with the character and any behavioral intentions that result from exposure to the story. The results show superior scores with the girls compared to the boys for all questions. Several aspects of the results discussed here, all of which merit further exploration, suggest a need to work on gender counter-stereotypes with children, but in different ways with girls than with boys. 


\section{INTRODUCTION}

En France, un Bulletin officiel de l'éducation nationale récent (BOEN $\left.\mathrm{n}^{\circ} 4 \mathrm{du} 22 / 01 / 2015\right)$ réaffirme le choix d'une politique éducative en faveur de l'égalité entre filles et garçons à l'école et se réfère à la « convention interministérielle pour l'égalité entre les filles et les garçons, les femmes et les hommes dans le système éducatif 2013-2018 ». Cette convention souligne la nécessité de travailler à la déconstruction des stéréotypes de sexe (notamment dans les ouvrages scolaires), de mettre en place des actions de décryptage des représentations stéréotypées filles-garçons dans les médias et de travailler à la diffusion des recherches sur le genre. L'éducation aux médias - permettant de développer l'esprit critique - et à la culture de l'égalité a ainsi, aujourd'hui, toute sa place dans les programmes scolaires de l'école élémentaire. Images et stéréotypes étant des concepts abordés en psychologie sociale (voir, par exemple, Moliner, 2016 ; McGarty, Yzerbyt, Spears, 2002), nous souhaitons nous intéresser à l'impact des contre-stéréotypes de sexe sur les enfants, dans le cadre d'une activité connue et régulière : la lecture d'albums de jeunesse.

\section{Stéréotypes et contre-stéréotypes de sexe}

Les stéréotypes, du point de vue psychosocial, sont considérés, ici, comme des croyances socialement partagées sur les caractéristiques personnelles (traits de personnalité, comportements, apparence...) des membres d'un même groupe (Leyens, Yzerbyt, Schadron, 1996 ; McGarty, Yzerbyt, Spears, 2002). Si les stéréotypes facilitent le quotidien de tous et se construisent à partir d'éléments de la réalité vécue (Wood, Eagly, 2010), rappelons que les stéréotypes de sexe sont composés, à la fois, d'éléments descriptifs - ce qui est typique d'un des sexes -, mais également prescriptifs - ce qui est souhaitable et attendu de la part d'un des sexes (Heilman, 2001).

Prolongeant cette réflexion, des recherches psychosociales ont été focalisées sur les contre-stéréotypes comme médium permettant de réduire les stéréotypes (Gawronski, Deutsch, Mbirkou, Seibt, Strack, 2008). En effet, les contre-stéréotypes sont les miroirs des stéréotypes socialement répandus, leur image exactement opposée (Finnegan, Oakhill, Graham, 2015). Dans le cas des contre-stéréotypes de sexe, si un comportement ou un trait de personnalité est considéré comme stéréotypique des hommes, il sera alors considéré comme contrestéréotypique des femmes. Les contre-stéréotypes peuvent représenter des transgressions envers les normes socialement partagées et, sortant des schémas prévisibles classiques, ils peuvent susciter un sentiment d'inconfort et d'incertitude (Flannigan, Miles, Quadflieg, Macrae, 2013). Cependant, certaines recherches, d'un point de vue sociocognitif, ont montré qu'en perturbant le processus habituel de catégorisation, les contre-stéréotypes inhiberaient en partie les connaissances antérieurement acquises et amèneraient ainsi les individus à moins stéréotyper (Goclowska, Crisp, 2013). Enfin, Gawronski et coll. (2008) ont mis en évidence qu'il était plus efficace de s'entraîner à l'affirmation de contre-stéréotypes positifs qu'à la négation de stéréotypes négatifs. Autrement dit, il serait plus efficace d'affirmer, par exemple, que « les filles sont courageuses » que d'affirmer que « les filles ne sont pas peureuses $»$.

Ainsi, considérant la force et la prégnance des stéréotypes de sexe du fait de leur caractère visible, mais, surtout, de leur fort ancrage social (Bussey, Bandura, 1999), une des possibilités et applications concrètes serait de ne pas se centrer directement sur leur déconstruction, mais de proposer de nouveaux réseaux d'associations via les contre-stéréotypes de sexe, peu étudiés encore à l'heure actuelle. Il est néanmoins important de souligner que ces derniers ne sont pas acceptés de la même manière par des observateurs selon qu'ils sont attribués ou réalisés par des hommes ou des femmes. En effet, l'asymétrie entre les sexes s'exemplifie par le fait que les contre-stéréotypes sont davantage acceptés pour les femmes que pour les hommes (Flannigan et coll., 2013 ; Tostain, Lebreuilly, 2006 ; Wilbourn, Kee, 2010). Déjà présents chez les enfants, les activités et domaines (jouets, métiers...) féminins sont moins valorisés, voire sont dévalorisés (Blakemore, 2003), dès le plus jeune âge, où les garçons sont davantage découragés d'avoir des comportements féminins (Dafflon Novelle, 2010). Dans notre étude, nous prolongeons ce questionnement sur la différence d'acceptation des contre-stéréotypes de sexe en nous y intéressant pour chaque catégorie de sexe.

\section{Des modèles d'identification possibles}

Les contre-stéréotypes sont portés par des individus, réels ou symboliques, qui pourront, selon les circonstances, devenir des modèles pour d'autres. Le modelage est une des formes les plus puissantes d'apprentissage (Bandura, 1977). Un modèle de réussite peut avoir des effets bénéfiques sur les performances, les attitudes et les comportements des individus observateurs, en leur permettant de se projeter eux-mêmes dans des situations similaires 
de réussite (Bagès, Martinot, Toczek, 2008). Marx et Roman (2002) identifient quatre critères nécessaires pour qu'une femme modèle ait un impact positif sur les performances d'étudiantes en mathématiques : faire partie d'un même groupe d'appartenance sociale (ici, les femmes), avoir un comportement que la cible estime être en capacité de réaliser, être perçue comme similaire aux niveaux physique et psychologique (ici, une femme ayant les mêmes intérêts académiques), être dans un domaine d'intérêt de l'observateur (ici, les mathématiques). Les auteurs ont également mis en évidence que l'effet bénéfique du modèle sur les performances des étudiantes n'était pas lié à sa présence physique, mais davantage à la perception de son expertise, de ses compétences dans le domaine d'intérêt. Bagès et coll. (2008) soulignent que cette expertise doit être expliquée par les efforts fournis par le modèle. De leur côté, Good, Woodzicka et Wingfield (2010) notent que la présentation conjointe de plusieurs modèles potentiels, hommes et femmes, permet aux filles et aux garçons d'avoir des performances similaires à des tests en sciences.

Dans une grande majorité de ces recherches, sont proposés des modèles, mais l'identification des individus à ces derniers, qui pourrait être un élément clé dans l'acceptation des contre-stéréotypes de sexe, n'est pas étudiée. Il est important de rappeler que les personnes présentant des attributs contre-stéréotypés ne sont pas forcément des modèles, bien au contraire, puisqu'elles transgressent les normes sociales. Un individu ne devient un modèle que lorsque les autres choisissent de l'imiter (Marx, Roman, 2002). Dans notre recherche, nous souhaitons ainsi nous intéresser, notamment, à la notion d'identification et de la place que celle-ci peut avoir dans le rapport aux contre-stéréotypes. Cette étude porte sur les personnages, modèles potentiels, présents dans la littérature de jeunesse, un agent périphérique de socialisation, tout comme le sont les jouets, habits, activités enfantines, médias (Dafflon Novelle, 2010).

\section{Un cas concret : la littérature pour enfants}

Un exemple de possibilité de présentation de modèles contre-stéréotypés, allant à l'encontre des normes sociales sexuées, est la littérature de jeunesse et, plus précisément, les albums à destination des enfants. Deux aspects particuliers sont alors à noter : le public spécifique auquel les albums s'adressent, les enfants et la spécificité du livre, la narration.

\section{Socialisation filles-garçons}

Les enfants connaissent, depuis leur naissance, une socialisation différenciée selon leur sexe, transversale à toute la société et déterminante dans le développement des rôles sociaux futurs (Dafflon Novelle, 2010 ; Rouyer, 2008). Les enfants ne sont pas éduqués, socialisés et pensés de la même manière selon qu'ils sont étiquetés filles ou garçons, dans les différents lieux de vie (famille, crèche, école, lieux péri et extra-scolaires...) et à travers les différents agents périphériques de socialisation (Dafflon Novelle, 2006). Cette socialisation différenciée participe, dès lors, à la construction de l'identité sexuée des enfants (Rouyer, 2007 ; Rouyer, Mieyaa, Le Blanc, 2014). Mieyaa et Rouyer (2013) se centrent sur la construction de l'identité sexuée du point de vue de la psychologie du développement et de la psychologie sociale. $\mathrm{Si}$ les enfants sont plus rigides face aux normes de sexe avant la « constance de genre » (atteinte aux environ de 6-7 ans), Rouyer (2007) décrit une période de flexibilité entre 7 et 12 ans, une phase de consolidation de l'identité sexuée et de compréhension que les conventions sociales, comme celles en lien avec les catégories de sexe, sont relatives.

\section{La littérature de jeunesse, un outil de transmission}

La littérature de jeunesse est inscrite dans les programmes d'enseignement (voir BOEN spécial $n^{\circ} 11$ du 26/11/2015) et décrite comme un médium permettant d'apprendre à lire, découvrir et comprendre le monde, développer des aptitudes à la réflexion critique, apprendre une langue étrangère... Il nous paraît alors important d'y porter attention et de s'intéresser aux modèles que les enfants peuvent rencontrer dans ces albums.

Les livres pour enfants sont un support privilégié d'acquisition des modèles sexués socialement définis (Brugeilles, Cromer, Cromer, 2002). Ce sont des narrations qui peuvent « transporter » les enfants et, grâce aux émotions développées envers les personnages, avoir un pouvoir persuasif entraînant une congruence avec les propos développés par l'histoire (Green, Brock, 2000 ; Mazzocco, Green, Sasota, Jones, 2010). Nous nous intéresserons à l'engagement émotionnel des enfants - le fait de ressentir des émotions pour et avec les personnages (Busselle, Bilandzic, 2009) -, lors de la lecture d'une histoire contre-stéréotypée. 


\section{OBJECTIFS ET HYPOTHÈSES DE L'ÉTUDE}

L'objectif de cette étude exploratoire est d'étudier l'impact de contre-stéréotypes de sexe présentés à travers des modèles auprès d'enfants de CE2-CM1, dans le cadre de la littérature de jeunesse. Cet impact est mesuré à l'aide d'un questionnaire - centré, d'une part, sur des éléments traduisant le lien avec le personnage (comme l'identification) et, d'autre part, sur des éléments intentionnels faisant suite à l'histoire (comme l'envie de réaliser la même activité contre-stéréotypée que le personnage). De manière générale, nous nous attendons à un effet différencié de cet impact selon le sexe des enfants et le niveau de stéréotypie de l'apparence physique du personnage illustré.

Notre première hypothèse porte sur les différences filles-garçons. Nous nous attendons à ce que, quelle que soit l'illustration (stéréotypée ou contre-stéréotypée) accompagnant l'histoire, les filles aient des scores significativement supérieurs aux garçons aux différentes questions posées aux enfants à travers le questionnaire (H1). Cette hypothèse est en lien avec l'asymétrie sociale et cognitive entre les sexes, où l'homme est la référence (Hurtig, Pichevin, 1986), la plus grande résistance des garçons face aux contre-stéréotypes de sexe (Pickering, Repacholi, 2001) et le fait qu'ils soient plus fortement découragés par leur entourage social lorsqu'ils adoptent des comportements « féminins » (Dafflon Novelle, 2010).

De plus, nous nous attendons à ce que les enfants, qui observent un personnage avec une apparence physique stéréotypée, aient des scores supérieurs aux items du questionnaire en comparaison des enfants observant un personnage avec une apparence contre-stéréotypée (H2). L'apparence physique est utilisée très précocement par les enfants dans le processus de catégorisation de sexe (Ndobo, 2010) - une personne avec une robe et des cheveux longs est une fille. Elle peut donc avoir une force et prégnance particulière. Considérant que l'histoire présentée aux enfants est contrestéréotypée, il nous semble que si le modèle est trop éloigné physiquement du quotidien des enfants, ils ne pourront pas s'identifier ni se projeter dans un comportement similaire.

\section{MÉTHODOLOGIE}

\section{Population}

La classe étant un milieu de vie sociale dont les enfants adoptent les règles et normes (Rouyer et coll., 2014), nous avons centré notre choix de population sur deux niveaux de classe de cycle 3 : le CE2 et le CM1. Cent vingt-sept enfants de CE2 et CM1, soit âgés de 8-9 ans, (53 CE2 et 74 CM1) ont participé à l'étude - 63 filles (25 CE2 et $38 \mathrm{CM} 1$ ) et 64 garçons (28 CE2 et $36 \mathrm{CM} 1$ ) - issus de deux écoles primaires situées dans la région urbaine lyonnaise. Les enfants ont été répartis de manière aléatoire dans les quatre conditions expérimentales, que nous exposerons dans la présentation des variables (tableau 1).

\begin{tabular}{lccc}
\hline & Filles & Garçons & Total \\
\hline $\begin{array}{l}\text { Apparence } \\
\text { stéréotypée }\end{array}$ & 31 & 34 & 65 \\
\hline $\begin{array}{l}\text { Apparence } \\
\text { contre-stéréotypée }\end{array}$ & 32 & 30 & 62 \\
\hline Total & 63 & 64 & 127 \\
\hline
\end{tabular}

Tableau 1. Répartition des participant.e.s dans les 4 conditions expérimentales selon le sexe des enfants et le type d'apparence phyisque du personnage observé.

\section{Procédure}

Les enfants, dont l'accord des parents a été sollicité préalablement, ont été réunis par groupes non mixtes de quatre ou cinq dans la bibliothèque de l'école, hors temps scolaire. Dans l'objectif de se rapprocher d'un contexte écologique, la passation collective a été choisie afin de recréer une situation connue, celle de la lecture « offerte » (Pasquier, 2014).

Une histoire, accompagnée d'une illustration, présentée pendant toute la durée de la passation, a été lue aux enfants, qui ont ensuite rempli individuellement le questionnaire. Lors des consignes introductives, il a été demandé aux enfants de ne pas réagir en collectif à l'histoire, afin que chacun puisse donner son avis par écrit. Pour finir, un temps de retour a été proposé avec des explications sur le protocole et la possibilité, pour les enfants, de faire des retours sur l'histoire entendue ou le questionnaire. La passation a duré entre 20 et 25 minutes.

\section{Matériel}

Les histoires contre-stéréotypées utilisées, de même que les illustrations, ont été créées pour cette recherche, afin d'éviter tout biais lié à leur reconnaissance. Ces créations sont fondées, notamment, sur une phase préalable, effectuée auprès de 11 enfants de 7-9 ans où il a été demandé : «Qu'est-ce que c'est une fille (garçon) ? Comment elle (il) est ? Qu'est-ce qu'elle (il) aime? ». Les enfants ont 
ensuite été invités à dessiner une fille et un garçon, ce qui a permis d'apporter des éléments sur l'apparence physique. S'agissant de contre-stéréotypes de sexe, les histoires (et les illustrations attenantes) sont différentes pour les filles et les garçons.

\section{Les histoires}

Les étapes (enfant pratiquant une activité contrestéréotypée et la présentant ensuite à ses camarades lors d'un exposé en classe avec un intérêt marqué de la part des autres élèves) et la longueur du récit sont identiques. Nous inspirant des éléments mentionnés par les enfants lors de la phase exploratoire, nous avons également fondé la création des histoires sur trois dimensions à travers lesquelles filles et garçons sont souvent opposés dans la littérature de jeunesse : intérieur-extérieur, privé-public et actifpassif (Dafflon Novelle, 2006). Ainsi, dans le cadre de contre-stéréotypes de sexe, le personnage fille grimpe dans les arbres, construit des cabanes et le personnage garçon écrit un journal intime dans sa chambre en y livrant ses émotions.

\section{Les illustrations}

Les quatre illustrations ont été créées en collaboration avec un artiste, par moitié avec le personnage fille et garçon - présentés en train de réaliser leur activité - où seule varie la stéréotypie de leur apparence physique. La fille stéréotypée a des cheveux longs blonds et porte une robe rose, tandis que la fille contre-stéréotypée a des cheveux courts châtains et porte un pantalon bleu. Le garçon stéréotypé a une coupe de cheveux courte et porte un pull avec un logotype similaire à ceux des marques vestimentaires, tandis que le garçon contre-stéréotypé a des cheveux plus longs, ondulés et porte un pull avec des fleurs vertes.

\section{Les variables}

\section{Variables indépendantes}

Nous avons manipulé l'apparence physique des personnages illustrés (stéréotypée ou contre-stéréotypée) et sélectionné une variable invoquée : le sexe des enfants.

\section{Variables dépendantes : le questionnaire}

Un questionnaire exploratoire de 6 items, mesurés à l'aide d'une échelle en six points, allant de " pas du tout d'accord » à « tout à fait d'accord »a été créé (tableau 2) :

- envie de faire la même activité que le personnage, afin de mesurer l'envie des enfants de réaliser eux-mêmes un comportement contre-stéréotypé ;
— sentiment de capacité à réaliser le comportement du personnage. La croyance en ses propres capacités joue un rôle pivot, notamment sur l'engagement futur des individus dans des comportements (Bussey, Bandura, 1999) ;

— lien émotionnel avec le personnage. L'histoire proposée est une forme de narration, qui peut induire un engagement émotionnel envers les personnages qui serait, lui-même, prédicteur de l'adhésion à l'histoire (Mazzocco et coll., 2010) ;

— identification/impression d'être le personnage. L'identification au personnage pourrait être un élément en lien avec l'acceptation de l'histoire contre-stéréotypée (Kwon, Lease, 2009) ;

— similitude avec le personnage, afin d'évaluer si les enfants se sentent similaires au personnage (Marx, Roman, 2002);

— souhait d'être l'ami du personnage. Le souhait d'être ami avec le personnage pourrait indiquer une certaine acceptation des comportements de ce dernier (Maisonneuve, 2004).

\begin{tabular}{ll}
\hline \multicolumn{2}{c}{ Items } \\
\hline 1 & $\begin{array}{l}\text { Cette histoire me donne envie de faire une } \\
\text { activité comme celle de Sarah (Adam). }\end{array}$ \\
\hline 2 & $\begin{array}{l}\text { Je me sens capable de faire la même chose que } \\
\text { Sarah (Adam). }\end{array}$ \\
\hline 3 & $\begin{array}{l}\text { À des moments de l'histoire, je ressentais la } \\
\text { même chose que Sarah (Adam). Quand elle (il) } \\
\text { était heureuse, j'étais heureuse aussi. }\end{array}$ \\
\hline 4 & $\begin{array}{l}\text { Quand j'écoutais, j'avais l'impression d'être le } \\
\text { personnage de l'histoire. }\end{array}$ \\
\hline 5 & Sarah (Adam) et moi, on est pareille. \\
\hline 6 & $\begin{array}{l}\text { J'aimerais être la copine de Sarah (copain } \\
\text { d'Adam). }\end{array}$ \\
\hline
\end{tabular}

Tableau 2. Questions posées aux enfants et mesurées à l'aide du échelle en 6 points

\section{RÉSULTATS}

Des MANOVA 2 (sexe des enfants) x 2 (stéréotypie du personnage) ont été réalisées pour les six dimensions.

\section{Variable «sexe des enfants»}

Les filles ont des scores significativement supérieurs à ceux des garçons, pour les 6 items de mesure : envie de réaliser la même activité $[F(1$, $123)=22,32, p<0,001]$, capacité à réaliser le même comportement $[F(1,123)=10,53, p=0,001]$, lien émotionnel $[F(1,123)=7,20, p=0,008]$, impression d'être le personnage $[F(1,123)=8,29, p=0,005]$, 


\begin{tabular}{|c|c|c|c|c|c|c|c|}
\hline & $\begin{array}{l}\text { Garçons } \\
\text { S }\end{array}$ & $\begin{array}{l}\text { Garçons } \\
\text { CS }\end{array}$ & $\begin{array}{l}\text { Total } \\
\text { garçons }\end{array}$ & $\begin{array}{l}\text { Filles } \\
\mathrm{S}\end{array}$ & $\begin{array}{l}\text { Filles } \\
\text { CS }\end{array}$ & $\begin{array}{l}\text { Total } \\
\text { filles }\end{array}$ & Total \\
\hline Envie de faire la même activité & $\begin{array}{l}3,15 \\
(1,78)\end{array}$ & $3,53(1,98)$ & $3,33(1,87)$ & $\begin{array}{l}5,32 \\
(1,17) \\
\end{array}$ & $\begin{array}{l}4,28 \\
(1,92)\end{array}$ & $\begin{array}{l}4,79 \\
(1,67)\end{array}$ & $\begin{array}{l}4,06 \\
(1,91)\end{array}$ \\
\hline $\begin{array}{l}\text { Capacité de réaliser la même } \\
\text { activité }\end{array}$ & $\begin{array}{l}3,88 \\
(2,09) \\
\end{array}$ & $3,87(1,93)$ & $3,88(2,00)$ & $\begin{array}{l}4,84 \\
(1,55) \\
\end{array}$ & $\begin{array}{l}4,97 \\
(1,49)\end{array}$ & $\begin{array}{l}4,90 \\
(1,51)\end{array}$ & $\begin{array}{l}4,39 \\
(1,84)\end{array}$ \\
\hline $\begin{array}{l}\text { Lien émotionnel avec le } \\
\text { personnage }\end{array}$ & $\begin{array}{l}2,79 \\
(1,61)\end{array}$ & $3,23(1,74)$ & $3,00(1,67)$ & $\begin{array}{l}4,16 \\
(1,53)\end{array}$ & $\begin{array}{l}3,50 \\
(1,95)\end{array}$ & $\begin{array}{l}3,83 \\
(1,77) \\
\end{array}$ & $\begin{array}{l}3,41 \\
(1,77)\end{array}$ \\
\hline Impression d'être le personnage & $\begin{array}{l}2,53 \\
(1,73)\end{array}$ & $3,03(1,96)$ & $2,77(1,84)$ & $\begin{array}{l}3,97 \\
(1,92) \\
\end{array}$ & $\begin{array}{l}3,47 \\
(1,72) \\
\end{array}$ & $\begin{array}{l}3,71 \\
(1,83) \\
\end{array}$ & $\begin{array}{l}3,24 \\
(1,89) \\
\end{array}$ \\
\hline Se sentir pareil au personnage & $\begin{array}{l}1,91 \\
(1,31) \\
\end{array}$ & $1,83(1,34)$ & $1,88(1,32)$ & $\begin{array}{l}3,71 \\
(1,83) \\
\end{array}$ & $\begin{array}{l}2,69 \\
(1,82)\end{array}$ & $\begin{array}{l}3,19 \\
(1,88) \\
\end{array}$ & $\begin{array}{l}2,53 \\
(1,74)\end{array}$ \\
\hline $\begin{array}{l}\text { Souhait d'être l'ami.e du } \\
\text { personnage }\end{array}$ & $\begin{array}{l}3,50 \\
(1,86)\end{array}$ & $4,37(1,73)$ & $3,91(1,84)$ & $\begin{array}{l}5,32 \\
(1,17)\end{array}$ & $\begin{array}{l}4,75 \\
(1,65)\end{array}$ & $\begin{array}{l}5,03 \\
(1,45)\end{array}$ & $\begin{array}{l}4,46 \\
(1,74)\end{array}$ \\
\hline
\end{tabular}

Tableau 3. Moyenne et écart-type des scores aux six questions selon le sexe des enfants et le type d'apparence physique du personnage observé.

similitude $[F(1,123)=21,95, p<0,001]$, souhait d'être l'ami du personnage $[F(1,123)=14,53$, $p<0,001$ ] (tableau 3 ).

Notre première hypothèse portant sur la différence de scores selon le sexe des enfants est ainsi validée.

\section{Variable «stéréotypie du personnage »}

Il n'y a pas de différence significative pour les scores des 6 questions selon la stéréotypie des personnages : envie de réaliser la même activité $[F(1,123)=1,12, p=0,29]$, capacité à réaliser le même comportement $[F(1,123)=0,03, p=0,86]$, lien émotionnel $[F(1,123)=0,13, p=0,72]$, impression d'être le personnage $[F(1,123)=0,00, p=0,99]$, similitude $[F(1,123)=3,78, p=0,054]$, souhait d'être l'ami du personnage $[F(1,123)=0,26$, $p=0,61]$. Notre deuxième hypothèse sur l'effet de la stéréotypie du personnage n'est donc pas validée : de manière générale, la variation de la stéréotypie de l'apparence physique des personnages n'a pas d'impact sur les réponses des enfants.

\section{Interaction entre le sexe des enfants et la stéréotypie du personnage}

L'interaction entre le sexe des enfants et la stéréotypie du personnage est significative pour deux questions : l'envie de réaliser la même activité que le personnage $[F(1,123)=5,32, p=0,02$, $\left.\eta_{p}^{2}=0,04\right]$ et le souhait d'être l'ami du personnage $\left[F(1,123)=6,19, p=0,01, \eta_{p}{ }^{2}=0,05\right]$. Pour l'envie de réaliser une activité similaire à celle du personnage, les tests post-hoc de Bonferroni montrent que les filles, ayant vu une illustration avec un personnage qui a une apparence stéréotypée $(M=5,32$,
$E T=1,17)$, déclarent significativement plus avoir envie de réaliser cette activité que les garçons, que ces derniers aient vu un personnage stéréotypé ( $M=$ $3,15, E T=1,78)(p<0,001)$ ou contre-stéréotypé $(M=3,53, E T=1,98)(p<0,001)$. Quant au souhait d'être l'ami du personnage, les tests post-hoc révèlent que les garçons, ayant vu le personnage stéréotypé, souhaitent significativement moins être l'ami du personnage $(M=3,50, E T=1,86)$ que les filles, et ce, qu'elles aient vu un personnage stéréotypé ( $M=$ $5,32, E T=1,17)(p<0,001)$ ou contre-stéréotypé $(M=4,75, E T=1,65)(p=0,01)$. L'interaction entre le sexe des enfants et la stéréotypie des personnages n'est pas significative pour les 4 autres questions : capacité à réaliser le même comportement $[F(1$, $123)=0,05, p=0,82]$, lien émotionnel $[F(1,123)=$ $3,27, p=0,07]$, impression d'être le personnage $[F(1,123)=2,38, p=0,13]$, similitude $[F(1,123)=$ $2,78, p=0,10]$ (tableau 3).

\section{DISCUSSION}

Cette étude exploratoire portait sur l'impact d'une histoire contre-stéréotypée sur des enfants de CE2-CM1, notamment à travers la variation de l'apparence physique du personnage illustré, l'objectif étant, alors, d'identifier d'éventuelles variables pertinentes, à travers le questionnaire, sur lesquelles travailler de manière plus approfondie dans des recherches ultérieures. Parmi les éléments originaux de cette recherche, la spécificité du public peut être soulignée : les enfants, des êtres en construction personnelle et sociale, auxquels il est important de s'intéresser, comme êtres actuels et non seulement comme des êtres en devenir (Danic, Delalande, Rayou, 2006). Si des évolutions 
concrètes en matière d'égalité femmes-hommes ont lieu (Gaborit, 2009), il apparaît nécessaire d'évaluer leur impact psychosocial, notamment en matière de stéréotypes, d'autant plus chez les enfants qui, selon cette évolution, devraient en être moins imprégnés. Un autre élément de cette étude à relever est l'intérêt porté aux concepts de modèles d'identification et de contre-stéréotypes de sexe à l'aide d'un outil peu étudié en psychologie sociale, celui de la littérature et, plus précisément, la littérature de jeunesse alliant, à la fois, contenus textuels et graphiques.

La première hypothèse de notre étude portait sur la différence de scores au questionnaire (destiné à mesurer l'impact de l'histoire) entre les filles et les garçons, après l'exposition à une histoire contrestéréotypée accompagnée d'une illustration, avec des scores supérieurs attendus chez les filles. Notre hypothèse est validée avec des scores significativement supérieurs chez les filles pour toutes les questions testées : envie de réaliser la même activité, capacité à réaliser le même comportement, lien émotionnel, impression d'être le personnage, similitude, souhait d'être l'ami du personnage.

Les garçons, ayant entendu l'histoire contrestéréotypée (où un garçon de leur âge écrit un journal intime), semblent être moins « entrés » dans l'histoire, avoir développé moins de liens avec le personnage et avoir moins envie de réaliser une activité similaire. Quant à la moindre sensibilité envers les comportements contre-stéréotypés de la part des garçons, ce résultat est en accord avec des observations antérieures (Wilbourn, Kee, 2010) : leur plus grande résistance aux contrestéréotypes (Peretz, 2016 ; Pickering, Repacholi, 2001) présente dès l'enfance (Rouyer, 2007). Nous pouvons, ici, rappeler la présence d'un large éventail de modèles offerts aux garçons, dès leur plus jeune âge et, notamment, dans la littérature de jeunesse (Brugeilles et coll., 2002 ; Pasquier, 2014) et dans l'enseignement scolaire de manière générale (Morge, Toczek, 2012). Ce constat, combiné à la présentation d'une histoire contre-stéréotypée, a pu participer au fait que l'histoire ait eu moins d'impact sur les garçons que sur les filles.

Il est également nécessaire de rappeler que notre étude présentait ces contre-stéréotypes à travers la littérature de jeunesse. Si nos résultats mettent en évidence un effet significatif du sexe sur les réponses aux questions sur l'histoire, il est important de prendre en considération le rapport différencié des filles et des garçons à la lecture, une activité majoritairement féminine (Pasquier, 2014), qui peut, en partie, expliquer les résultats obtenus, indiquant un moindre engagement des garçons dans l'histoire.

Dans un second temps, compte tenu de la place précoce que tient l'apparence physique dans le processus de catégorisation de sexe (Ndobo, 2010) et l'aspect essentiel de l'apparence physique genrée dans la construction du genre (Halim et coll., 2014), nous nous attendions à ce que celle-ci ait un impact sur la réception de l'histoire. Cependant, notre seconde hypothèse, portant sur la stéréotypie de l'apparence physique des personnages illustrés, n'est pas validée : les scores aux questions sur l'histoire ne varient pas, que les enfants aient vu le personnage représenté avec une apparence physique stéréotypée ou contre-stéréotypée. Comme nous avions pu le mentionner précédemment, l'apparence physique est rarement étudiée en tant que domaine des stéréotypes de sexe (Miller et coll., 2009). Dans leur recherche, ils ont d'ailleurs mis en évidence un rapport différencié des enfants qu'ils ont interrogés, par rapport aux normes d'apparence physique selon le sexe : des stéréotypes sur l'apparence physique, plus accessibles chez les filles que chez les garçons et une utilisation plus fréquente de descripteurs d'apparence pour décrire une fille qu'un garçon. Ces résultats nous amènent à penser que la non-validation de notre hypothèse sur la stéréotypie de l'apparence physique peut être mise en relation avec ce rapport différencié à l'apparence. Pour ces raisons, nous nous intéresserons aux effets d'interaction mis en évidence dans notre étude.

Deux interactions significatives entre le sexe des enfants et la stéréotypie de l'apparence physique du personnage ont été mises en évidence. La première a trait à l'envie de réaliser le même comportement (contre-stéréotypé) et la seconde au souhait d'être l'ami du personnage. Ces interactions mettent en évidence deux phénomènes distincts, que nous allons analyser séparément.

Les filles ayant vu le personnage stéréotypé, se démarquent significativement des garçons - qu'ils aient vu un personnage stéréotypé ou contre-stéréotypé - en souhaitant davantage réaliser une activité similaire à celle du personnage, c'est-à-dire une activité contre-stéréotypée. Si les tests post-hoc réalisés n'ont pas mis en évidence de différences significatives de scores entre les filles ayant vu un personnage d'apparence stéréotypé ou contre-stéréotypé, nous pouvons cependant penser que l'apparence physique stéréotypée tient une place particu- 
lière pour les filles, comme nous avons pu l'exposer précédemment en nous fondant, notamment, sur les travaux de Halim et coll. (2014) ainsi que sur ceux de Miller et coll. (2009). Un personnage ayant une apparence stéréotypée, mais réalisant une activité contre-stéréotypée, pourrait être un moyen, pour les filles, de se projeter dans un comportement similaire. La petite fille grimpe dans les arbres avec sa robe. Aucune tenue particulière n'est nécessaire. Ainsi, toutes peuvent le faire si elles le souhaitent. Il serait intéressant d'approfondir cette hypothèse et de s'intéresser au point de vue des filles. La place de l'apparence physique, pour les filles, nous apparaît comme un axe de recherche à poursuivre. En effet, il se pourrait que la présence d'éléments stéréotypés, notamment dans l'apparence, fournisse des repères connus, pouvant modérer l'incertitude et l'anxiété provoquées par les contre-stéréotypes (Flannigan et coll., 2013). Dans notre cas, la littérature de jeunesse apparaît comme un moyen pertinent de sensibiliser les filles aux contre-stéréotypes et, dans une optique plus concrète, il se pourrait que les albums « non sexistes » présentant souvent des personnages stéréotypés ou neutres au niveau de leur apparence soient une manière pertinente de sensibiliser les filles à des comportements contrestéréotypés.

La deuxième interaction significative a trait au souhait d'être l'ami du personnage. Dans ce cas, ce sont les garçons, ayant vu le personnage avec une apparence stéréotypée, qui souhaitent significativement moins être l'ami du personnage que les filles (qu'elles aient vu un personnage stéréotypé ou contre-stéréotypé). De la même manière que précédemment, bien que les tests post-hoc n'aient pas mis en évidence de différence de scores, pour le souhait d'être l'ami du personnage, entre les garçons ayant vu le personnage stéréotypé et ceux l'ayant vu contre-stéréotypé, la question de l'amitié et de l'apparence physique nous semblent être un élément à approfondir du côté des garçons. L'amitié est fondée, très souvent, sur une homophilie, un « attrait du même » (Maisonneuve, 2004). La réalisation graphique permettant une réelle distinction entre le personnage stéréotypé et contre-stéréotypé n'a pas été aisée, les caractéristiques vestimentaires - de même que les autres éléments attenants à l'apparence : coiffure, bijoux, chaussures - étant notamment moins diversifiées pour les garçons que pour les filles (Dafflon Novelle, 2006). De ce fait, le personnage stéréotypé (coupe courte, gel dans les cheveux, vêtements de marque) a pu être perçu comme un garçon «populaire », qui, de surcroît, écrit un journal intime (une activité pouvant être assimilée au domaine scolaire « féminin »). Considérant l'amitié comme une relation d'égalité et de réciprocité (Poulin, 2012), il est possible que le personnage ait été perçu comme trop éloigné des garçons ayant entendu l'histoire et n'ait pas entraîné l'envie pour ces derniers de projeter une amitié avec ce personnage, même imaginaire.

\section{Limites et perspectives}

Marquant l'intérêt de travailler sur la littérature de jeunesse de par sa place importante au sein du système scolaire français, mais également de par la combinaison texte/illustration qu'elle offre, il serait intéressant de réaliser une étude comprenant une condition « sans illustration », afin, justement, d'en mesurer l'impact - image décrite comme porteuse de messages (Moliner, 2016). De même, considérant les moindres scores des garçons aux différentes questions, il pourrait être intéressant de proposer des conditions « histoire stéréotypée » et « histoire contre-stéréotypée », afin de savoir si les garçons entrent effectivement moins dans l'histoire de manière globale et, ainsi, de comprendre l'impact réel d'une histoire contre-stéréotypée sur cette population. Une phase plus qualitative pourrait ensuite permettre de saisir ce que pensent alors les garçons de ces deux histoires très différentes. Ces deux dispositifs possibles pourraient être évalués à plus long terme, prenant en considération le fait que les enfants peuvent ne pas être habitués à de telles histoires sortant des schémas narratifs traditionnels et que des expositions répétées peuvent être nécessaires à l'acceptation de comportements contrestéréotypés. Comme l'expliquent Finnegan et coll. (2015), des expositions fréquentes à des contre-stéréotypes devraient permettre de rendre ces associations contre-stéréotypées plus accessibles et, ainsi, participer à la réduction des stéréotypes.

La question de l'exposition plus fréquente à ce type d'ouvrages peut également être mise en lien avec la place essentielle que joue l'entourage social des enfants dans la construction de leur identité sexuée (Mieyaa, Rouyer, 2013). L'école est un lieu de vie sociale, ainsi que d'apprentissage des rôles, normes et valeurs (Mosconi, 2004). Si la « convention interministérielle pour l'égalité entre les filles et les garçons, les femmes et les hommes dans le système éducatif 2013-2018» est une étape nécessaire pour tendre vers plus d'égalité, il ne faut pas omettre la place essentielle que jouent les acteurs et les actrices sociaux en contact quotidien avec les enfants : les enseignants. S'intéressant plus préci- 
sément à la littérature de jeunesse comme médium permettant de sensibiliser les élèves à l'égalité, Marro, Pasquier et Breton (2016) ont proposé un guide permettant aux enseignants de sensibiliser les enfants aux inégalités, en sollicitant leurs émotions. Notre étude a porté sur l'impact d'albums de jeunesse contre-stéréotypés, mais des recherches, comme celle précédemment citée, apparaissent essentielles dans une prise en compte globale de ce que vivent les enfants dans le cadre du développement de leur identité sexuée.

\section{CONCLUSION}

Un des points essentiels, que met en évidence notre étude, est la nécessité d'approfondir et de développer des recherches autour des contre-stéréotypes de sexe auprès des enfants, en portant une attention particulière à la fois aux filles et aux garçons. S'agissant plus précisément les filles, la présence d'éléments stéréotypiques associés à l'endogroupe pourrait être un élément clé à prendre en compte dans les recherches et actions ayant pour but de favoriser l'ouverture aux contre-stéréotypes. Il pourrait être intéressant, dans une perspective de recherches futures, de tenter d'autres croisements entre éléments stéréotypés et contre-stéréotypés.
Du côté des garçons, notre étude a montré une moindre sensibilité aux contre-stéréotypes de leur part, qu'il convient d'approfondir. Si une grande majorité des études sur les contre-stéréotypes de sexe et les modèles a trait aux filles et aux femmes (Peretz, 2016), il est nécessaire de penser des recherches similaires auprès des garçons, sous peine de soutenir l'asymétrie entre les sexes, la valorisation des activités et métiers " masculins » et de perpétuer la «fabrique des garçons » (Ayral, 2014). De même, comme nous y invite Tostain (2016a), il est essentiel de ne pas s'intéresser exclusivement aux stéréotypes de sexe dans le cadre de méthodologies expérimentales. Porter une attention particulière sur les stéréotypes en milieu écologique permet de prendre en compte les individus (dans notre cas les enfants), les contextes sociaux réels et les variabilités existant à l'intérieur même d'une catégorie de sexe (Tostain, 2016b). Nous avons pu voir, dans la présente étude, que l'amitié est un élément qu'il convient d'approfondir. Aussi, les amitiés de garçons étant vécues davantage en groupe par rapport aux filles (Benenson, Heath, 2006), le groupe peut avoir un impact important sur les garçons et pourrait être un élément clé vers plus d'ouverture aux contre-stéréotypes de sexe.

\section{RÉFÉRENCES}

Ayral (Sylvie).- Introduction, dans Ayral (S.), Raibaud (Y.), Pour en finir avec la fabrique des garçons. Volume 1, Pessac, Maison des sciences de l'homme d'Aquitaine, 2014, p. 15-33.

Bagès (Céline), Martinot (Delphine), Toczek (MarieChristine).- Le rôle modérateur de l'explication donnée à la réussite d'un modèle féminin sur la performance des filles en mathématiques : une étude exploratoire, Cahiers internationaux de psychologie sociale, 80, 2008, p. 3-11.

BAndurA, (Albert).- Social learning theory [1977], trad. fr., L'apprentissage social, Bruxelles, Mardaga, 1980.

Benenson (Joyce F.), Heath (Anna).- Boys withdraw more in one-on-one interactions, whereas girls withdraw more in groups, Developmental Psychology, 42, 2, 2006, p. 272-282.

Blakemore (Judith E. W.).- Children's beliefs about violating gender norms: Boys shouldn't look like girls, and girls shouldn't act like boys, Sex Roles, 48, 2003, p. 411-419.
Brugeilles (Carole), Cromer (Isabelle), Cromer (Sylvie)-- Les représentations du masculin et du féminin dans les albums illustrés ou comment la littérature enfantine contribue à élaborer le genre, Population, 57, 2, 2002, p. 261-292.

Busselle (Rick), Bilandzic (Helena).- Measuring narrative engagement, Media Psychology, 12, 4, 2009, p. 321-347.

Bussey (Kay), BANDuRA (Albert).-- Social cognitive theory of gender development and differentiation, Psychological Review, 106, 4, 1999, p. 676-713.

DAFFlon Novelle (Anne).- D'avant à maintenant, du bébé à l'adulte : synthèse et implications de la socialisation différenciée des filles et des garçons, dans Dafflon Novelle (A.), Filles-garçons. Socialisation différenciée, Grenoble, Presses universitaires de Grenoble, 2006, p. 27-54.

Dafflon Novelle (Anne).- Pourquoi les garçons n'aiment pas le rose? Pourquoi les filles préfèrent Barbie à Batman? Perception des codes sexués et construction 
de l'identité sexuée chez des enfants âgés de 3 à 7 ans, dans Rouyer (V.), Croity-Belz (S.), Prêteur (Y.), Genre et socialisation de l'enfance à l'âge adulte : expliquer les différences, penser l'égalité, Toulouse, Érès, 2010, p. 25-40.

Danic (Isabelle), Delalande (Julie), Rayou (Patrick). Enquêter auprès d'enfants et de jeunes. Objets, méthodes et terrains de recherche en sciences sociales, Rennes, Presses universitaires de Rennes, 2006.

Finnegan (Eimear), OAKhill (Jane), Garnham (Alan).Counter-stereotypical pictures as a strategy for overcoming spontaneous gender stereotypes, Frontiers in Psychology, 6, 2015, 1291.

Flannigan (Natasha), Miles (Lynden K.), Quadflieg (Susanne), Macrae (C. Neil).- Seeing the unexpected: Counterstereotypes are implicitly bad, Social Cognition, 31, 6, 2013, p. 712-720.

GABORIT (Pascaline).- Les stéréotypes de genre : identités, rôles sociaux et politiques publiques, Paris, L'harmattan, 2009.

Gawronski (Bertram), Deutsch (Roland), Mbirkou (Sawsan), Seibt (Beate), Strack (Fritz).- When "just say no" is not enough: Affirmation versus negation training and the reduction of automatic stereotype activation, Journal of Experimental Social Psychology, 44, 2, 2008, p. 370-377.

GoclowsKa (Małgorzata A.), CRISP (Richard J.).-- On counter-stereotypes and creative cognition: When interventions for reducing prejudice can boost divergent thinking, Thinking Skills and Creativity, 8, 2013, p. 72-79.

Good (Jessica J.), Woodzicka (Julie A.), Wingfield (Lylan C.).- The effects of gender stereotypic and counterstereotypic textbook images on science performance, The Journal of Social Psychology, 150, 2, 2010, p. 132-147.

Green (Melanie C.), Brock (Timothy C.).- The role of transportation in the persuasiveness of public narratives, Journal of Personality and Social Psychology, 79, 5, 2000, p. 701-721.

Halim (May Ling), Ruble (Diane N.), Tamis-LeMonda (Catherine. S.), Zosuls (Kristina M.), Lurye (Leah E.), Greulich (Faith K.).- Pink frilly dresses and the avoidance of all things "girly": Children's appearance rigidity and cognitive theories of gender development, Developmental Psychology, 50, 4, 2014, p. 1091-1101.

Heilman (Madeline E.).- Description and prescription: How gender stereotypes prevent women's ascent up the organizational ladder, Journal of Social Issues, 57, 2001, p. 657-674.

Hurtig (Marie-Claude), Pichevin (Marie-France).- La différence des sexes, Paris, Tierce, 1986.

Kwon (Kyongboon), Lease (A. Michele).- Perceived influence of close friends, well-liked peers, and popular peers reputational or personal influence?, Journal of Social and Personal Relationships, 31, 8, 2014, p. 11161133.

Leyens (Jacques-Philippe), Yzerbyt (Vincent), SchA-
DRON (Georges).-- Stéréotypes et cognition sociale, Wavre, Mardaga, 1996.

Maisonneuve (Jean).- Psychologie de l'amitié, Paris, Presses universitaires de France, 2004.

Marro (Cendrine), Pasquier (Gaël), Breton (Laurence).- Les ressentis émotionnels, une entrée pour éduquer à l'égalité des sexes et à la littérature à l'école primaire, Tréma, 46, 2016, p. 77-88.

Marx (David M.), Roman (Jasmin S.).- Female role models: Protecting women's math test performance, Personality and Social Psychology Bulletin, 28, 9, 2002, p. 1183-1193.

Mazzocco (Philip J.), Green (Melanie C.), Sasota (Jo A.), Jones (Norman W.).- This story is not for everyone: Transportability and narrative persuasion, Social Psychological and Personality Science, 1, 2010, p. 361-368.

McGarty (Craig), Yzerbyt (Vincent), Spears (Russel).- Stereotypes as Explanations. Cambridge, Cambridge University Press, 2002.

Mieyaa (Yoan), Rouyer (Véronique).- Genre et socialisation de l'enfant : pour une approche plurifactorielle de la construction de l'identité sexuée, Psychologie française, 58, 2013, p. 135-147.

Miller (Cindy F.), Lurye (Leah E.), Zosuls (Kristina M.), Ruble (Diane. N.).- Accessibility of gender stereotype domains: Developmental and gender differences in children, Sex Roles, 60, 11-12, 2009, p. 870-881.

Moliner (Pascal).- Psychologie sociale de l'image, Grenoble, Presses universitaires de Grenoble, 2016.

Morge (Ludovic), Toczek (Marie-Christine).Connaître les modalités d'expression des stéréotypes de genre pour éviter leur transmission : le cas des situations d'entrée des séquences d'investigation, dans Calmettes (B.), Didactique des sciences et démarches d'investigation : références, représentations, pratiques et formation, Paris, L'Harmattan, 2012, p. 95-118.

Mosconi (Nicole).- De l'inégalité des sexes dans l'éducation familiale et scolaire, Ville, école, intégration, 138, 2004, p. 15-22.

NDobo (André).- Les nouveaux visages de la discrimination, Bruxelles, De Boeck, 2010.

PAsquier (Gaël).- Enseigner l'égalité des sexes par la littérature de jeunesse à l'école primaire : quelle place pour les garçons ?, dans Ayral (S.), Raibaud (Y.), École, loisirs, sports, culture : la fabrique des garçons, Bordeaux, Maison des sciences de 1'Homme d'Aquitaine, 2014, p. 161-184.

Peretz (Tal).-- Why Study Men and Masculinities? A Theorized Research Review, Graduate Journal of Social Science, 12, 3, 2016, p. 30-43.

Pickering (Samantha), Repacholi (Betty).-- Modifying children's gender-typed musical instrument preferences: The effects of gender and age, Sex Roles, 45, 9-10, 2001, p. 623-643.

Poulin (François).- Recherches actuelles sur les relations entre pairs, dans Lemelin (J.-P.), Provost (M.A.), 
Tarabulsy (G. M.), Plamondon (A.), Dufresne (C.) (Eds), Développement social et émotionnel chez l'enfant et l'adolescent. Les bases du développement. Tome 1, Québec, Presses de 1'Université du Québec, 2012, p. 313-354.

ROUYER (Véronique).- Enfance, dans Rouyer (V.), La construction de l'identité sexuée, Paris, Armand Collin, 2007, p. 113-124.

ROUYER (Véronique).- La construction de l'identité sexuée du point de vue de la psychologie du développement et de la psychologie sociale, Neuropsychiatrie de l'enfance et de l'adolescence, 56, 6, 2008, p. 335-338.

Rouyer (Véronique), Mieyaa (Yoan), Le Blanc (Alexis).- Socialisation de genre et construction des identités sexuées, Revue française de pédagogie, 187, 2, 2014, p. 97-137.

Tostain (Manuel), Lebreuilly (Joëlle).- Liens entre flexibilité des rôles de sexe et explications de l'origine des différences entre sexes : études chez des enfants de 5 à 11 ans et des adultes, Bulletin de psychologie, 59, 6, 2006, p. 615-627.

Tostain (Manuel).- Faut-il en finir avec les stéréotypes de sexe ? Revue de questions critique sur les études psychosociales des relations entre sexes, Bulletin de psychologie, 69, 3, 2016a, p. 163-178.

Tostain (Manuel).- Pour en finir avec la domination masculine ? Regard critique sur les approches psychosociales des relations entre sexes, Bulletin de psychologie, 69, 5, 2016b, p. 345-363.

Wilbourn (Makeba Parramore), KeE (Daniel W.).Henry the nurse is a doctor too: Implicitly examining children's gender stereotypes for male and female occupational roles, Sex Roles, 62, 9-10, 2010, p. 670-683.

Wood (Wendy), EAGLy (Alice H.).- Gender, dans Fiske (S. T.), Gilbert (D. T.), Lindzey (G.), Handbook of social psychology, Hoboken, John Wiley, 2010, p. 629-667. 